\title{
La competencia digital de los estudiantes universitarios latinoamericanos
}

\author{
Digital literacy of Latin American university students
}

\author{
Jorge Ángel Martínez Navarro. \\ Universitat Rovira i Virgili. \\ ajorgemartinez@gmail.com
}

\section{RESUMEN.}

La investigación que se presenta es un primer acercamiento al estudio del nivel de competencia digital autopercibida de los estudiantes universitarios en el contexto latinoamericano con una muestra representativa de 5 países de la región.

Para la recogida de datos se ha utilizado el instrumento INCOTIC adaptado para el contexto Latinoamericano, obteniendo datos por edad y sexo sobre la alfabetización Informacional (DAI), tecnológica (DAT), multimedia (DAM) y comunicativa (DAC).

En los resultados se detecta la DAI más alta se da en el rango de edad entre los 24-31 años, y la DAT, DAM y DAC se da en el rango de 28-31 años. En el caso de las mujeres, la DAI más alta se da en el rango de edad entre los 20-27 años, y la DAT, DAM y DAC se da en el rango de 28-31 años.

Como conclusión se observa que el país con menor puntuación en las alfabetizaciones analizadas es Ecuador, siendo Chile y Colombia los países con mejor puntuación. Se observan profundas diferencias al profundizar por género y edad en cada alfabetización.

Se espera diseñar las líneas maestras de un Plan de Alfabetización Digital para estudiantes universitarios con propuestas para cada una de las universidades participantes.

\section{PALABRAS CLAVE.}

Competencia digital, Educación Superior, alfabetización tecnológica, género, edad.

\section{ABSTRACT.}

This research is a first approach to the study of the self-perceived digital competence of Latin American students with a broad sample of 5 countries in the region.

For the collection of data we have used INCOTIC instrument adapted to the Latin American context, obtaining data by age and sex on Information Literac (DAI). technological literacy (DAT), multimedia literacy (DAM) and communicative literacy (DAC).

Specifically, the highest DAI is in the age range between 24-31 years, and the DAT, DAM and DAC is in the range of 28-31 years. In the case of women, the highest DAl is in the age range between 20-27 years, and DAT, DAM and DAC are in the range of 28-31 years.

To sum up, the country with the lowest score obtained in the analyzed literacies is Ecuador, with Chile and Colombia being the countries with the highest score. In addition, interesting differences are observed when deepening by gender and age in each literacy.

It is expected to design the guidelines of a Digital Literacy Plan for university students with proposals for earch university that will be the input for researchers from each country.

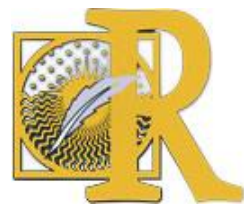

Fecha de recepción: 23-10-2019 Fecha de aceptación: 04-11-2019

Martínez-Navarro, J. A. La competencia digital de los estudiantes universitarios latinoamericanos International Journal of Educational Research and Innovation (IJERI), 14, 276-289 ISSN: 2386-4303 DOI https://doi.org/10.46661/ijeri.4387 


\section{KEY WORDS.}

Digital Literacy, Higher Education, Technological Literacy, gender, age.

\section{Introducción.}

En el estudio de la competencia digital de los estudiantes universitarios latinoamericanos predominan los acercamientos descriptivos y de corto alcance en cuanto a las poblaciones estudiadas, es escasa la investigación dirigida a evaluar la competencia, y cuando se hace se emplean instrumentos diseñados a propósito debido a la ausencia de estándares propios. Respecto al estudio de la competencia digital de los estudiantes universitarios latinoamericanos, predominan los acercamientos descriptivos y de corto alcance en cuanto a las poblaciones estudiadas; y es escasa la investigación dirigida a evaluar la competencia, y cuando se hace se emplean instrumentos diseñados a propósito debido a la ausencia de estándares propios.

Recientemente, Castellano, Sánchez y Calderero (2017) concluyen en su investigación que los alumnos universitarios que participaron en su investigación no comparten los rasgos que se esperan de un nativo digital: en especial en lo que se refiere a producir, difundir y consumir cultura a través de Internet. Por tanto, podría hablarse de una brecha digital no por falta de acceso o de uso sino más bien por ausencia de competencia digital.

La ausencia de un nivel óptimo de competencia digital en los estudiantes universitarios latinoamericanos debe ser vista por el sistema educativo como un reto importante. Dicen Gutiérrez y Tyner (2012) que "Lo más preocupante sería que la escolaridad obligatoria no cumpliese su función básica de alfabetizar, entendida ésta como preparación para la vida en la sociedad digital".

La evaluación de la competencia digital de los estudiantes se hace imprescindible en este escenario, pues un adecuado diagnóstico aportará información de base para las propuestas de alfabetización que se diseñen e implementen desde el sistema educativo. Esta investigación pretende partir de INCOTIC1 como instrumento que mide la autopercepción de competencia digital en estudiantes universitarios, lo adecúa al español que se habla en Latinoamérica y convoca la participación de cinco países de América del Sur (Ecuador, Colombia, Perú, Chile y Venezuela) para aportar un perfil aproximado de la competencia digital auto percibida por el estudiante sudamericano.

\section{Marco teórico.}

La interacción de los estudiantes con las tecnologías de la Información y la Comunicación (TIC) permite el desarrollo de conocimientos, habilidades y destrezas de forma integrada, es así como el aprendiz digital es aquella generación nacida a partir de los años 80 (al menos muchas de sus características) por el hecho de crecer en el contexto digital y tecnológico la generación Net (Prensky, 2001; Palfrey \& Gasser, 2008).

Las TIC han originado nuevos retos en todos los ámbitos de la sociedad, creando la necesidad de replantear el sistema educativo (Comisión Europea, 2012; OECD, 2012,), por ello, de una manera progresiva se han ido involucrando en los distintos niveles educativos, desde la educación infantil hasta la universitaria, y desde hace ya varios años atrás, se comenzó a incorporar en las aulas materiales con soporte informático y a implementar el

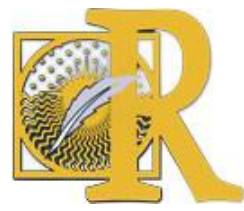

Fecha de recepción: 23-10-2019 Fecha de aceptación: 04-11-2019

Martínez-Navarro, J. A. La competencia digital de los estudiantes universitarios latinoamericanos

International Journal of Educational Research and Innovation (IJERI), 14, 276-289

ISSN: 2386-4303 DOI https://doi.org/10.46661/ijeri.4387 
su uso con la intención de preparar al alumnado para afrontar de forma reflexiva y crítica la información que recibe por diferentes medios y desarrollar las nuevas competencias.

Con las TIC no solo se logra innovar en las aulas, sino que se han originado transformaciones y replanteamientos en los procesos de enseñanza-aprendizaje, en donde el rol del docente y del alumno. El docente se ha convertido en el facilitador de experiencias y el alumno es el protagonista de la construcción de su propio aprendizaje (Comisión Europea, 2012 \& 2013; UNESCO, 2008 \& 2011; Losada y García, 2018; Núñez, et al. 2019; Moliní y Sánchez, 2019).

\subsection{Diferencias entre Nativos Digitales e Inmigrantes Digitales.}

Para Prensky (2010) reconocer la diferencia entre el nativo digital y el inmigrante digital es fundamental para poder establecer los planes necesarios que permitan la instrucción, de esta manera se logra establecer que el nativo digital desde que nace ha tenido la oportunidad de interactuar con la tecnología siendo está su práctica y medio cotidiano, mientras que el inmigrante digital, es aquel que ha tenido que aprender a relacionarse con la tecnología con posterioridad, por lo que aplica las estrategias que ha adquirido sin tecnología, pero incorporando las TIC. Con esto, los nativos digitales según piensan y procesan la información de diferente manera a los inmigrantes digitales, teniendo un lenguaje digital que estos últimos no tienen (Esteve, Duch, y Gisbert, 2014).

Autores como Blanco (2014) ponen en duda que crecer en un entorno 2.0 signifique saber desempeñarse de manera adecuada en el mismo, y refiere que son muchos los alumnos universitarios que pasan muchas horas ante el ordenador, pero no saben cómo obtener información ni usar los contenidos y tampoco se muestran interesados en ellos.

Estos resultados demuestran que la competencia digital de la que tanto se habla en la literatura científica, puede ser inferior a la de los inmigrantes digitales y, al respecto, se pronuncian investigadores como Gallardo-Echenique et al., (2016). Hacer una diferenciación entre los nativos digitales y los inmigrantes digitales para Kennedy et al. (2010), es dejar a un lado la posibilidad de que entre unos y otros, en este caso estudiantes y profesores, tengan habilidades y experiencias, que puedan combinarse para el manejo de las TIC en distintas circunstancias educativas. Tal y como lo refieren Bennett, Maton y Kervin (2008) no hay pruebas suficientes de que haber nacido y crecido en la era digital otorgue a los jóvenes estilos de aprendizaje diferentes a los que ya se tenían.

\subsection{La competencia digital de los estudiantes universitarios.}

Según Coll (2008) las TIC descubren nuevas opciones al desarrollo de la enseñanza debido a que usan elementos didácticos que propician: el aprender a aprender, la enseñanza cooperativa, el incentivo, las habilidades tecnológicas y la investigación del conocimiento en diversas áreas.

El Informe Horizon (Alexander et al., 2019) evidencia el crecimiento de las oportunidades de los estudiantes para que combinen su educación formal con cursos modulares en línea, a un precio asequible; respondiendo a las necesidades de los estudiantes que desean un mayor control sobre las vías de aprendizaje al obtener un certificado o un título. Además, se indica que son muchos los estudiantes que actualmente confían en sus dispositivos móviles como

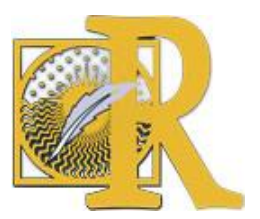

Fecha de recepción: 23-10-2019 Fecha de aceptación: 04-11-2019

Martínez-Navarro, J. A. La competencia digital de los estudiantes universitarios latinoamericanos International Journal of Educational Research and Innovation (IJERI), 14, 276-289 ISSN: 2386-4303 DOI https://doi.org/10.46661/ijeri.4387 
herramientas de su proceso de enseñanza-aprendizaje, permitiendo acceder a los contenidos en cualquier momento y lugar.

Una vez presentadas la importancia de la inclusión de las TIC en la Educación, las características de la generación digital y la competencia digital de los estudiantes universitario, se pretende realizar un estudio del nivel de competencia digital autopercibida de los estudiantes universitarios en el contexto latinoamericano, teniendo como variables objeto de estudio: la alfabetización Informacional, la alfabetización tecnológica, la alfabetización multimedia y la alfabetización comunicativa; analizando los datos por países y comparando los resultados para cada uno de ellos en función de la edad y el género.

\section{Parte empírica.}

Respecto al estudio de la competencia digital de los estudiantes universitarios latinoamericanos predominan los acercamientos descriptivos y de corto alcance en cuanto a las poblaciones estudiadas, es escasa la investigación dirigida a evaluar la competencia, y cuando se hace se emplean instrumentos diseñados a propósito debido a la ausencia de estándares propios mencionada también para el caso de la CDD.

Recientemente Castellano, Sánchez y Calderero (2017) concluyen en su investigación que los alumnos universitarios que participaron en su investigación no comparten los rasgos que se esperan de un nativo digital: en especial en lo que se refiere a producir, difundir y consumir cultura a través de Internet. Por tanto, podría hablarse de una brecha digital no por falta de acceso o de uso sino más bien por ausencia de competencia digital.

La ausencia de competencia digital en los estudiantes universitarios latinoamericanos debe ser vista por el sistema educativo como un reto importante. Dicen Gutiérrez y Tyner (2012) que "Lo más preocupante sería que la escolaridad obligatoria no cumpliese su función básica de alfabetizar, entendida ésta como preparación para la vida en la sociedad digital" (p. 36). La evaluación de la competencia digital de los estudiantes se hace imprescindible en este escenario, pues un adecuado diagnóstico aportará información de base para las propuestas de alfabetización que se adelanten desde el sistema educativo.

\section{Objetivos.}

El objetivo general de este trabajo es evaluar, de manera comparada entre países, el nivel de competencia digital autopercibida de los estudiantes universitarios latinoamericano.

Para ello se ha utilizado un instrumento diseñado, validado y fiabilizado en el contexto español y del cual se ha realizado una adaptación para el contexto Latino Americano (con su correspondiente validación). Con este instrumento se evaluará competencia digital de los estudiantes, y esta avaluación aportará información para realizar propuestas de alfabetización posteriores.

El objetivo específico de esta investigación se centra en evaluar los niveles de competencia digital autopercibida. Planteándose las siguientes preguntas de investigación:

- ¿Cómo es la alfabetización digital autopercibida en cada uno de los países?

- Dentro de esa alfabetización digital, ¿tienen los estudiantes igual de desarrolladas todas las componentes: ¿tecnología, multimedia...?

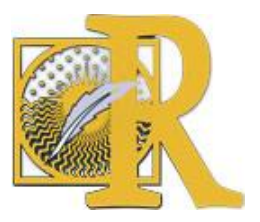

Fecha de recepción: 23-10-2019 Fecha de aceptación: 04-11-2019

Martínez-Navarro, J. A. La competencia digital de los estudiantes universitarios latinoamericanos International Journal of Educational Research and Innovation (IJERI), 14, 276-289 ISSN: 2386-4303 DOI https://doi.org/10.46661/ijeri.4387 
- Dentro del rango de edad de los estudiantes, ¿afecta la edad en los resultados obtenidos?

- ¿ ¿Tienen la misma puntuación los estudiantes de ambos sexos, o existe diferencia?

- ¿ ¿Existe algún patrón en el rango de edad de los estudiantes al relacionarlo con sus puntuaciones en cada una de las alfabetizaciones?

\section{Metodología.}

La investigación se realizará bajo enfoque cuantitativo, de tipo descriptivo. En cuanto a su pretensión, se busca de acuerdo con Hernández, Collado y Baptista (2015) caracterizar el objeto de estudio, en este caso haciéndolo por contraste con los estándares establecidos en competencia digital. Su alcance es exploratorio, pues a la fecha no se ha evaluado la competencia digital autopercibida en estudiantes universitarios de Latinoamérica con un mismo instrumento y una muestra amplia.

El instrumento seleccionado para la recogida de datos será la herramienta INCOTIC, diseñado por González y otros (2012) del grupo de investigación ARGET de la URV; el cual, ha sido probado en varios estudios en el contexto español en la ESO y en la universidad.

Para la elaboración del cuestionario, se partió de las reflexiones generales de Storey (2002) acerca de la usabilidad de las herramientas TIC y de las competencias C2 y C3 aprobadas por la URV. Respecto a la estructura del mismo, en la primera parte hay tres secciones: recoger los datos de identificación de los informantes, recoger los recursos digitales de los que dispone y el grado real de uso de las TIC en general. La segunda parte del cuestionario proporciona datos para valorar la competencia inicial en TIC del alumnado.

La fiabilidad del instrumento seleccionado se puede considerar excelente tras los análisis de fiabilidad correspondientes, obteniendo valores para la Alfa de Cron Bach de 0.914 para el índice de actitudes y de 0.913 para la competencia digital (González-Martínez et al., 2018). Además de este análisis estadístico de la fiabilidad, INCOTIC ya había experimentado un proceso de validación de instrumentos clásico, basado en diferentes fases de revisión con panel de expertos y pilotaje (Gisbert et al., 2011).

Previamente a este trabajo se han realizado las fases de adaptación y puesta en línea de la herramienta INCOTIC para Latinoamérica, así como la administración del cuestionario a la muestra de estudiantes seleccionados en los cinco países. Para eso, se ha recurrido a un experto lingüista que realizo los ajustes semánticos necesarios y adecuando el vocabulario. La versión preliminar se aplicada a una muestra de informantes voluntarios (muestra piloto) que verificaron la correcta comprensión de los conceptos y del sentido de las preguntas que se realizan.

En este trabajo nos centraremos en los datos obtenidos sobre la alfabetización Informacional (DAI), que se centra en evaluar la capacidad de acceder, gestionar, evaluar y la información al servicio del aprendizaje, la tecnológica (DAT) centrada en evaluar si existe obstáculos en la gestión del software o del hardware, la Multimedia (DAM), que evalúa la capacidad de creación y comprensión de contenidos multimedia, y la Comunicativa (DAC), que evalúa el modo de comunicación con herramientas digitales.

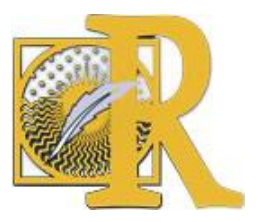

Fecha de recepción: 23-10-2019 Fecha de aceptación: 04-11-2019 


\section{INTERNATIONAL JOURNAL OF EDUCATIONAL RESEARCH AND INNOVATION

Todos los indicadores analizados son de tipo Likert 1 a 5 (Nada (no sé hacerlo), Poco (no sé si podría llegar a conseguirlo), Algo (es posible que pudiera conseguirlo), Bastante (creo que podría llegar a conseguirlo), Totalmente (sé hacerlo) y "0: no sabe no contesta".

\section{Población y muestra.}

La población de este estudio está constituida por estudiantes universitarios de América del Sur. El muestreo se ha realizado en dos etapas. En la primera fase se usó el muestreo por conveniencia para escoger las universidades que participarían en el estudio de acuerdo a los siguientes criterios:

- Universidades públicas y privadas.

- Universidades grandes, mediana y pequeñas.

- Universidades que tengan facultad del área social y del aérea de ciencias exactas. Se seleccionó una muestra de quince universidades que cumplían estos criterios en cinco países de América del Sur. La distribución por países es como sigue:

Tabla 1. Población. Alumnos de las universidades participantes

\begin{tabular}{|l|l|l|l|}
\hline País & Universidad & Alumnos & $\%$ \\
\hline Chile & Santiago & 21.793 & $8,7 \%$ \\
\hline Chile & Adolfo Bañez & 2.289 & $0,9 \%$ \\
\hline Chile & Viña del Mar & 2.819 & $1,1 \%$ \\
\hline Colombia & Bucaramanga & 5.062 & $2,0 \%$ \\
\hline Colombia & Fco de Paula & 15.366 & $6,2 \%$ \\
\hline Colombia & Industrial de Santander & 20.451 & $8,2 \%$ \\
\hline Perú & Católica & 21.957 & $8,8 \%$ \\
\hline Perú & Ciencias Aplicadas & 44.270 & $17,8 \%$ \\
\hline Ecuador & ULEAM & 14.242 & $5,7 \%$ \\
\hline Ecuador & UNACH & 8.563 & $3,4 \%$ \\
\hline Ecuador & PUCESI & 2.389 & $1,0 \%$ \\
\hline Ecuador & UTPL & 51.831 & $20,8 \%$ \\
\hline Venezuela & Los Andes & 22.044 & $8,8 \%$ \\
\hline Venezuela & UCAB & 15.088 & $6,1 \%$ \\
\hline Venezuela & Monte Ávila & 1.183 & $0,5 \%$ \\
\hline & TOTAL & $\mathbf{2 4 9 . 3 4 7}$ & \\
\hline
\end{tabular}

Viendo los datos de la tabla superior se observa que existe una descompensación entre el número de sujetos de la muestra por universidades, ya que la implicación de todas las universidades no ha sido la misma. El caso más favorable, es el de Ecuador, donde la universidad de la IP del proyecto, quiso que todos sus estudiantes de primer curso contestaran el cuestionario. De este modo, en el caso de la Universidad de Ecuador disponemos de la población total y en el resto de países solo tenemos una muestra. El total de participantes que cumplimentó el instrumento fue de 5.344.

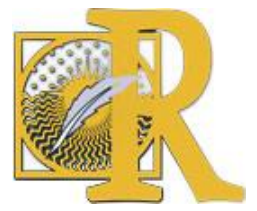

Fecha de recepción: 23-10-2019 Fecha de aceptación: 04-11-2019 
Tabla 2. Comparativa de población y tamaño de muestra por países.

\begin{tabular}{|l|l|l|l|}
\hline País & Muestra & Población & $\%$ \\
\hline Ecuador & 3.438 & 77.025 & $4,46 \%$ \\
\hline Colombia & 273 & 40.879 & $0,67 \%$ \\
\hline Perú & 768 & 66.227 & $1,16 \%$ \\
\hline Chile & 327 & 26.901 & $1,22 \%$ \\
\hline Venezuela & 538 & 38.315 & $1,40 \%$ \\
\hline & & & \\
\hline Total & 5.344 & 249.347 & $2,14 \%$ \\
\hline
\end{tabular}

\section{Resultados.}

Como parte inicial del análisis de los datos, dado el volumen inicial de los mismo se realizó una fase de limpieza y depuración de los datos, quedando sólo los campos con los que se iban a trabajar en esta investigación. Posteriormente, dado que uno de los campos era la edad, y que había un rango muy amplio de edades (entre 16 y 87 años), se realizaron agrupaciones. Estas agrupaciones en rangos de edad son inicialmente de cuatro años, aumentando hasta llegar a diez años para el rango de edades entre 70 y 79 años, permitiendo así obtener unas conclusiones más detalladas en los años comprendidos entre los 16 y 35 años. Por último, se realizó un análisis estadístico de los datos, estableciendo la media y desviación de los mismo.

Los tipos de alfabetización que fueron estudiadas son: informacional (DAI), alfabetización Tecnológica (DAT), alfabetización Multimedia (DAM) y la alfabetización Comunicativa (DAC), las cuales fueron estudiadas en los países latinoamericanos (Chile, Colombia, Ecuador, Perú y Venezuela) y separado por género (mujer y hombre) obteniendo los gráficos comparativos Gráfico 1 y Gráfico 2.

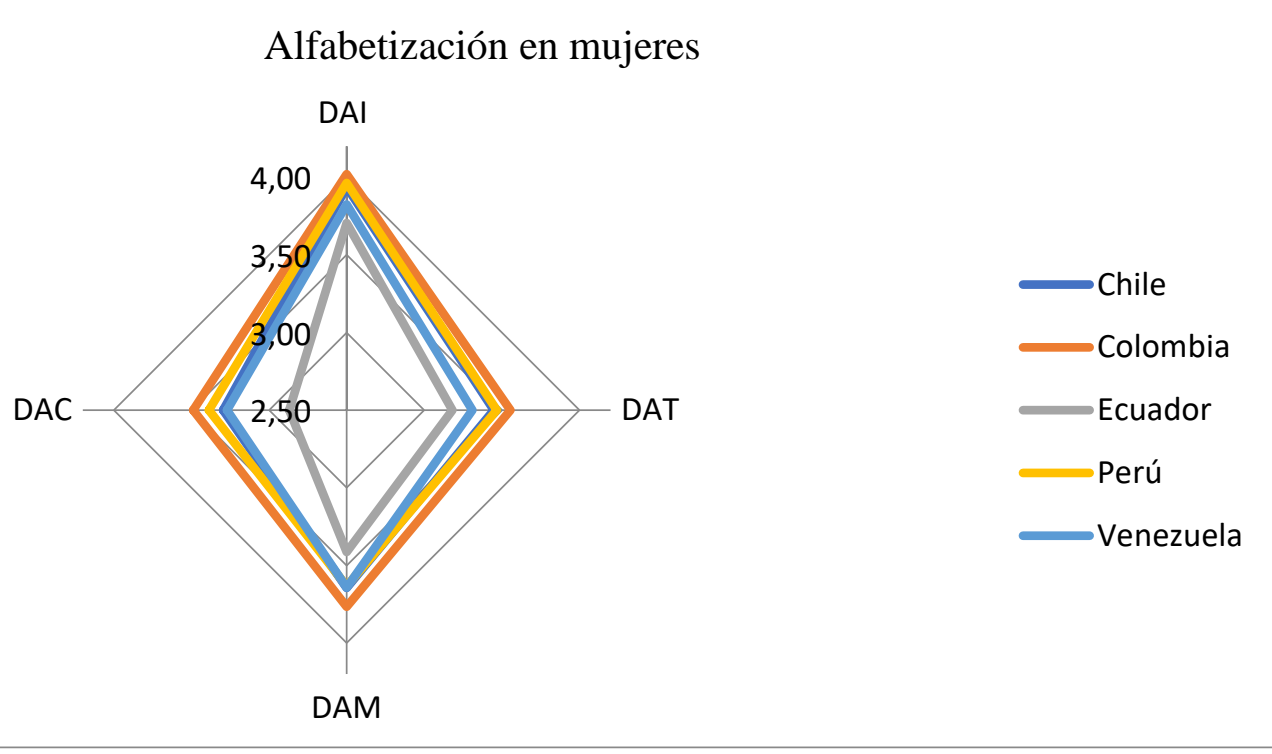

Gráfico 1. Comparativa por países del grado de alfabetización. Hombres.

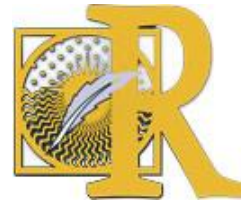

Fecha de recepción: 23-10-2019 Fecha de aceptación: 04-11-2019 
Alfabetización en Hombres

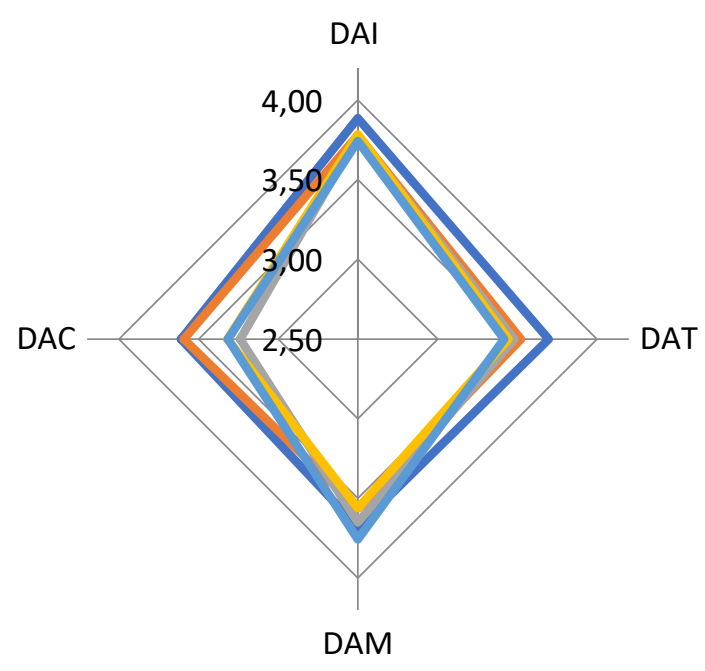

Chile

Colombia

Ecuador

$\longrightarrow$ Perú

Venezuela

Gráfico 2. Comparativa por países del grado de alfabetización. Mujeres.

- $\quad$ Alfabetización informacional (DAl): En Chile la media de utilización de los recursos informativos es la más alta con respecto al resto de los países de estudio alcanzando una media 3,94 en mujeres y 3,89 en hombres. El país con la media más baja es Ecuador con 3,71 en mujeres y 3,78 en hombres. En cuanto a la evaluación por género las mujeres tienen una media más alta en la utilización de las herramientas informacionales en edades comprendidas de los 20 a los 27 años, siendo Colombia el país con un porcentaje más alto. En el caso de los hombres la utilización o el acceso a la información es más frecuente en edades comprendidas desde los 24 hasta los 31 años, los países con la media más alta para el género masculino es Colombia y Venezuela.

- $\quad$ Alfabetización Tecnológica (DAT): Chile es el país con la media más alta en utilización de las herramientas tecnológicas las mujeres tiene una media de 3,45 y los hombres 3,70 . El país con la media más baja en la utilización de las herramientas tecnológicas es Ecuador, las mujeres con 3,31 y los hombres con una media de 3,44. En función al estudio efectuado por género y por edades, las mujeres de edades comprendidas entre los 28 y 31 años son las que tienen acceso a la alfabetización tecnológica y el país con mayor variación es Colombia. Con respecto al género masculino, se observa la media más alta en el mismo renglón de edad y el país con la mayor variación es también Colombia.

- $\quad$ Alfabetización Multimedia (DAM): La media de utilización más alta de los países de estudio en la alfabetización multimedia es para Venezuela seguido de Colombia, con una media de 3,70 donde las mujeres tienen una media de 3,64 y los hombres 3,76. La media más baja para el uso de las herramientas multimedia la tiene Ecuador. Las

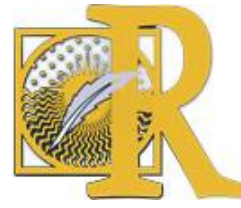

Fecha de recepción: 23-10-2019 Fecha de aceptación: 04-11-2019 
mujeres con 3,41 y los hombres con 3,65. En el estudio separado para observar el comportamiento por genero se observa que en las mujeres de edad comprendida de los 28 a los 31 años, siendo el país con la media más alta Colombia con 4,47; en el caso de los hombres de igual manera las edades entre 28 y 31 años con una media de 3,81 y Colombia continua con la media de utilización de herramientas más altas en la distribución por género.

- $\quad$ Alfabetización Comunicativa (DAC): La media más alta en los países de estudio para el área de alfabetización comunicativa la tiene Colombia con una media de 3,54; donde las mujeres tienen una media de 3,49 y los hombres 3,59. El país con la media más baja es Ecuador donde la media de las mujeres es de 2,87 y la de los hombres se ubica en 3,32. En cuanto a la evaluación estadísticas de las medias de utilización de las herramientas comunicacionales tenemos que las mujeres en edades comprendidas entre los 28 y los 31 años tiene la media más alta y por género femenino Colombia es el país con una media 4,60. En cuanto a los hombres las edades comprendidas entre los 28 y 31 años es la media más alta de utilización con 3,78, continúa Colombia con las medias altas en utilización de esta herramienta.

\section{Conclusiones.}

Una vez analizados los datos, hay que dar solución a las preguntas de investigación planteadas en el punto 3.1. Como conclusiones podemos destacar que los países en los que los estudiantes universitarios tienen mayor nivel en las cuatro alfabetizaciones con una media más elevadas son Chile y Colombia, mientras que las más bajas se encuentran en Ecuador y Perú, obteniendo una desviación típica similar para los cinco países. Respecto a la utilización de las herramientas informativas, tecnológicas, multimedia y comunicativas hasta la edad de los 28 destacar que en el caso de las mujeres es equidistante o superior a la utilización de los recursos, mientras que los hombres muestran curvas crecientes que se mantienen hasta alcanzar edades maduras superiores a los 50 años.

Individualmente, en cada una de las alfabetizaciones, destaca que hay un único país que es el que tiene las medias más bajas en las cuatro alfabetizaciones, que es Ecuador. En contraposición, en DAI y DAT el país con la media más alta es Chile, en DAM es Venezuela, y en DAC es Colombia.

Si realizamos ahora un análisis comparado por género y rango de edad, podemos observar como en el caso de los hombres, la DAI más alta se da en el rango de edad entre los 24-31 años, y la DAT, DAM y DAC se da en el rango de 28-31 años. En el caso de las mujeres, la DAl más alta se da en el rango de edad entre los 20-27 años, y la DAT, DAM y DAC se da en el rango de 28-31 años.

Además de esto, en los cinco países se da un rango de edad en el que las cuatro alfabetizaciones son sustancialmente más bajas, siendo en el caso de Chile entre los 36 y 39 años, en el caso de Ecuador entre 40 y 59 años, y de 32 a 35 años en el de Perú.

Las conclusiones para Ecuador, que es el país que tiene mayor tamaño de la muestra, podemos destacar las siguientes conclusiones. En DAI no hay diferencias entre los estudiantes de ambos sexos, no ocurriendo así en las otras tres alfabetizaciones donde si hay grandes diferencias: siendo del $9,4 \% 6.9 \%$ y $12.8 \%$ superiores en el caso de los hombres

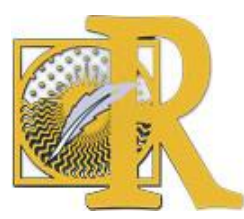

Fecha de recepción: 23-10-2019 Fecha de aceptación: 04-11-2019

Martínez-Navarro, J. A. La competencia digital de los estudiantes universitarios latinoamericanos International Journal of Educational Research and Innovation (IJERI), 14, 276-289 ISSN: 2386-4303 DOI https://doi.org/10.46661/ijeri.4387 
para las alfabetizaciones DAT, DAM y DAC respectivamente. En edades menores de 31 años las diferencias por sexo en estas 3 últimas alfabetizaciones son considerablemente menores que en rangos superiores.

Siguiendo con Ecuador, la DAI se mantiene constante en un rango de edad muy amplio [16-49] en ambos sexos, y es a partir de esa edad cuando se empiezan a producir grandes diferencias. En el caso de DAT, en el mismo rango de edades se aprecia un ligero incremento de la alfabetización para ambos sexos conforme aumenta el rango de edad. Con DAM ocurre al contrario ya que en ese mismo rango, en este caso se aprecia un ligero decremento de la alfabetización siendo más acusado en las mujeres. Por último, en DAC y en el rango de edades [16-49] se aprecia solo un decremento en el caso de las mujeres.

Como se ha comentado previamente, en los cinco países, los estudiantes que menos tiempo llevan en la universidad tienen en todas las alfabetizaciones (DAM, DAC, DAT y DAI), puntuaciones más bajas que los del rango de 32 a 35 años, y es que a medida que el contexto se ha digitalizado más, la competencia digital de los estudiantes mejora porque llegan a la universidad con otras habilidades TIC (más amplias y mejores) comprobándose como el haber nacido y crecido en la era digital otorgue a los jóvenes estilos de aprendizaje diferentes a los que ya se tenían ( Bennett, Maton y Kervin (2008)).

Con esto observamos que la competencia digital es más alta en los alumnos en el rango de edad de 32 a 35 años que los nativos digitales comprobándose como no siempre los más jóvenes son los que autoperciben más nivel de CD, obteniendo así las mismas conclusiones Castellano, Sánchez y Calderero (2017) donde los universitarios que participaron en su investigación no compartían los rasgos que se esperan de un nativo digital: en especial en lo que se refiere a producir, difundir y consumir cultura a través de Internet. Por tanto, podría hablarse de una brecha digital no por falta de acceso o de uso sino más bien por ausencia de competencia digital.

La ausencia de competencia digital en los estudiantes universitarios latinoamericanos debe ser vista por el sistema educativo como un reto importante. Dicen Gutiérrez y Tyner (2012) que "Lo más preocupante sería que la escolaridad obligatoria no cumpliese su función básica de alfabetizar, entendida ésta como preparación para la vida en la sociedad digital" (p. 36). La evaluación de la competencia digital de los estudiantes se hace imprescindible en este escenario, pues un adecuado diagnóstico aportará información de base para las propuestas de alfabetización que se adelanten desde el sistema educativo.

Todas estas conclusiones realizadas se basan en los resultados obtenidos a través del estudio de la autopercepción, es importante contrastar estos datos y conclusiones con una evaluación real de la alfabetización digital a través de un instrumento dedicado para ello, de modo que se pueda comprobar si los resultados son similares.

Los resultados, aun siendo muy descriptivos son coincidentes con otras investigaciones como la de Castellano, Sánchez y Calderero (2017), donde concluían que los nativos digitales no tenían alfabetizaciones más altas frente a los inmigrantes digitales.

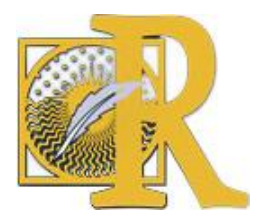

Fecha de recepción: 23-10-2019 Fecha de aceptación: 04-11-2019 


\section{Propuestas.}

Con estas conclusiones, las propuestas a realizar para cada una de las universidades de cada país serían las siguientes.

Para mejorar aquellas alfabetizaciones en las que el nivel de autopercepción es menor es necesario incrementar la formación complementaria de los estudiantes universitarios en las herramientas digitales; $y$ es que, como se ha podido comprobar el hecho de que éstos utilicen a diario aplicaciones de entretenimiento y comunicación, no implica que tengan las competencias digitales necesarias.

\section{Países que han tenido resultados bajos.}

Estas recomendaciones habría que hacerlas especialmente a las universidades de Ecuador ya que es el país que ha obtenido las menores medias para las cuatro alfabetizaciones (destacando las diferencias encontraras el caso de la DAC en las mujeres). El alumnado no cuenta con las competencias digitales necesarias de modo que se tienen que reforzar para estar al nivel de los otros países de Latinoamérica analizados, y compartir los rangos que se esperan de un nativo digital. Especialmente habría que proponer cursos de formación para la mejora de la alfabetización comunicativa, que permita que los alumnos se desenvuelvan con soltura para acciones básicas como el montaje de videocurrículums, el envío de ficheros grandes, realizar videoconferencias a tres, etc.

\section{Países que han tenido resultados altos.}

Las universidades de Chile y Colombia, que son las que mejores resultados han obtenido en todas las alfabetizaciones, deberían centrarse en la mejora de la alfabetización comunicativa y tecnológica que son las que tienen menores resultados. Es por esto, que además de los cursos que se han recomendado previamente para el caso de las universidades de Ecuador, sus estudiantes deberían realizar cursos de formación de ofimática para que aprendan tareas básicas como el envío de emails con copia oculta, diseñar plantillas personalizadas en las presentaciones...

Las universidades de Chile y Colombia han obtenido resultados similares, y al haber obtenido los niveles más altos en las alfabetizaciones analizadas, deben ser conscientes que tienen los niveles más altos de Latinoamérica obteniendo una mejora de su autoestima y que no se han apreciado diferencias entre hombres y mujeres.

\section{Países que han tenido resultados medios.}

Estas últimas recomendaciones son también válidas para las universidades de Perú y Venezuela, ya que son las universidades que han obtenido resultados intermedios y tienen también como alfabetizaciones más bajas DAC y DAT.

El alumnado de las universidades de Perú y Venezuela están en un escalón inferior a las anteriores, proponiendo a las mismas, que hay que reforzar estas alfabetizaciones para poder acceder, gestionar, evaluar y la información al servicio de sus aprendizajes para enfrentarse con éxito a los retos de la Sociedad del Conocimiento en el contexto de los estudios universitarios.

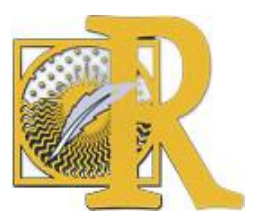

Fecha de recepción: 23-10-2019 Fecha de aceptación: 04-11-2019 
Si nos centramos en el análisis por rango de edad, las universidades de Chile deberían reforzar los cursos de formación de mejora de las 4 alfabetizaciones, para los alumnos del rango de edad comprendido entre los 36 y 39 años, ya que son los que han presentado las puntuaciones más bajas. En el caso de Ecuador, el rango de edad para el que más habría que fomentar los cursos de las cuatro alfabetizaciones sería de 40 a 59 años en el caso de Ecuador, y de 32 a 35 años en el de Perú.

\section{Recomendaciones generales para todos.}

En general, todas las universidades deberían crear un programa general para promover el uso de las TIC en el proceso de aprendizaje de los estudiantes y un programa específico para mejorar la alfabetizacion digital de las mujeres hasta obtener resultados similares a los hombres.

\section{Bibliografía}

- Adell, J. (1997). Tendencias en educación en la sociedad de las tecnologías de la información. EDUTEC, Revista Electrónica de Tecnología Educativa, 7, 1-21.

- Ashour, S. (2019). How technology has shaped university students' perceptions and expectations around higher education: an exploratory study of the United Arab Emirates. Studies in Higher Education, 1-13.

- Aviram, A. \& Eshet-Alkalai, Y. (2006). Towards a theory of digital literacy: three scenarios for the next steps. European Journal of Open, Distance and E-learning, 1, 1-11.

- Baelo, R. y Cantón, I. (2009). Las tecnologías de la información y la comunicación en la educación superior. Estudio descriptivo y de revisión. Revista Iberoamericana de Educación, 50(7).

- Bennett, S., Maton, K., \& Kervin, L. (2008). The digital natives debate: A critical review of the evidence. British Journal of Educational Technology, 39(5), 775-786.

- Blanco, S. (2014). Nativos digitales y tecnoanalfabetos funcionales. Cibersur.com. En: http://www.cibersur.com/016018/nativos/digitales/tecnoanalfabetos/funcionales

- Boase, J. (2013). Implications of software-based mobile media for social research. Mobile Media \& Communication, 1(1), 57-62. doi:10.1177/2050157912459500

- Bullen, M., Morgan, T., Belfer, K. \& Qayyum, A. (2009). The net generation in higher education: Rhetoric and reality. International Journal of Excellence in E- Learning, 2(1), 1-13.

- Burgos Aguilar, J. V., \& Lozano Rodríguez, A. (2011). Tecnología educativa y redes de aprendizaje de colaboración. Retos y realidades de innovación en el ambiente educativo.

- Claro, M., Preiss, D. D., Martín, E. S., Jara, I., Hinostroza, J. E., Valenzuela, S.,Nussbaum, M. (2012). Assessment of 21st century ICT skills in chile: Test design and results from high school level students.

- Covello, S. (2010). A review of Digital Literacy Assessment Instruments. IDE-712 FrontEnd Analysis Research. Analysis for Human Perfonmance Technology Decisions.

- Duncan-Howell, J. A., \& Lee, K. T. (2007). M-Learning: Innovations and initiatives: Finding a place for mobile technologies within tertiary educational settings. Ascilite 2007, Singapore.

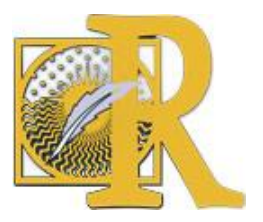

Fecha de recepción: 23-10-2019 Fecha de aceptación: 04-11-2019 
- Echenique, E. E. G. (2013). Hablemos de estudiantes digitales y no de nativos digitales. Universitas Tarraconensis. Revista de Ciències de l'Educació, 1(1), 7-21.

- Esteve, F., Adell, J., \& Gisbert, M. (2013). El laberinto de las competencias clave y sus implicaciones en la educación del siglo XXI. Il congreso internacional multidisciplinar de investigación educativa. Tarragona, 4-5 Julio.

- Esteve, F. M., Duch, J., \& Gisbert, M. (2014). Los aprendices digitales en la literatura científica: Diseño y aplicación de una revisión sistemática entre 2001 y 2010. Pixel-Bit, Revista de Medios y Educación, 45, 9-21.

- Fajardo, I., Villalta, E. y Salmerón, L. (2016). ¿Son realmente tan buenos los nativos digitales? Anales de psicología,32(1), 89-97.

- Gallardo-Echenique, EE, Marqués -Molías, L., Bullen, M. y Strijbos, JW (2016). Hablemos de aprendices digitales en la era digital. Revista mexicana de bachillerato a distancia, 8(15), 148-181.

- García, I., Peña-López, P., Johnson, L., Smith, R., Levine, A., \& Haywood, K. (2010). Informe Horizon: Edición iberoamericana 2010. Austin, Texas: The New Media Consortium.

- Gisbert, M. y Esteve, F. (2016). Digital Learners: la competencia digital de los estudiantes universitarios. La Cuestión Universitaria, 7, 48-59.

- Gisbert, M. \& Lázaro, JL. (2014). L'avaluació de la competència digital docent. 28 Jornada DIM. Barcelona.

- González-Martínez, J., et al. (2018). INCOTIC 2.0. Una nueva herramienta para la autoevaluación de la competencia digital del alumnado universitario. Profesorado 22 (4), 133-152.

- Henríquez, P., Moncada, G., Chacón, L., Dallos, J., \& Ruiz, C. (2012). Nativos digitales: aproximación a los patrones de consumo y hábitos de uso de internet, videojuegos y celulares. Revista Educación y Pedagogía, 24(62), 145-156.

- Hobbs, R. (2010). Digital and Media Literacy: A Plan of Action. Whashington, DC: The Aspen Institute.

- Johnson, L., Adams Becker, S., Estrada, V., \& Freeman, A. (2014). NMC Horizon Report: 2014 Higher Education Edition. Austin, Texas: The New Media Consortium.

- Johnson, L., Adams, S., \& Cummins, M. (2012). NMC Horizon Report: 2012 Higher Education Edition. Austin, TX: The New Media Consortium.

- Jorgensen, B. (2003). Baby boomers, generation $X$ and generation Y?: Policy implications for defence forces in the modern era. Foresight, 5(4), 41- 49.

- Kennedy, G., Judd, T., Dalgarno, B., \& Waycott, J. (2010). Beyond natives and immigrants: exploring types of net generation students. Journal of Computer Assisted Learning, 26(5), 332-343.

- Lenhart, A., Rainie, L., \& Lewis, O. (2001). Teenage life online: The rise of the instantmessage generation and the internet's impact on friendship and family relations. Pew Internet \& American Life Project.

- Mansell, R., \& Tremblay, G. (2013). Renewing the knowledge societies vision for peace and sustainable development. Paris: United Nations Educational, Scientific and Cultural Organization (UNESCO).

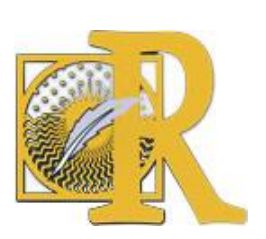

Fecha de recepción: 23-10-2019 Fecha de aceptación: 04-11-2019 
- Marqués, P. (2000). Impacto de las TIC en la enseñanza universitaria. Facultad de Educación Universidad Autónoma de Barcelona.

- Martínez Bonafé, J. (2012). El problema del conocimiento en el triángulo entre capitalismo, crisis y educación. Investigación En La Escuela, 76, 7-22.

- McCrindle, M. (2006). New generations at work: Attracting, recruiting, retaining and training generation $Y$. The ABC of $X Y Z$.

- Moya, A. (2009). Las nuevas tecnologías en la educación. Innovación y experiencias educativas, 24(1), 1-9.

- Oblinger, D., \& Oblinger, J. L. (2005). Educating the net generation (Vol. 264).Educause Washington, DC.

- OCDE. (2005). La definición y selección de competencias clave. Resumen ejecutivo. Organización para la Cooperación y el Desarrollo Económico.

- Organización de las Naciones Unidas ONU. (febrero de 2016). Transversalización de la Agenda 2030 para el Desarrollo Sostenible. de https://undg.org/wpcontent/uploads/2017/03/UNDG-Mainstreaming-the-2030-Agenda-Reference-

Guide_Spanish-clean.pdf

- Palfrey, J. \& Gasser, U. (2008). Born digital: Understanding the first generation of digital natives. New York: Basic Books.

- Pérez Gómez, A. I. (2007). La naturaleza de las competencias básicas y sus aplicaciones pedagógicas. Consejería de Educación. Gobierno de Cantabria. Cuadernos de Educación de Cantabria.

- Prensky, M. (2010). Nativos e inmigrantes digitales. Distribuidora Sek.

- Sánchez, J., Ruíz, J., Sánchez, E., (2017). Flipped classroom. Claves para su puesta en práctica. Revista de Educación Mediática y TIC. 6 (2):336-358

- Selwyn, N. (2011). Education and technology. Key issues and debates. London: Continuum.

- Somerville, M. M., Lampert, L. D., Dabbour, K. S., Harlan, S., \& Schader, B. (2007). Toward large scale assessment of information and communication technology literacy: Implementation considerations for the ETS ICT literacy instrument. Reference Services Review, 35(1), 8-20. doi:10.1108/00907320710729337

- Uceda, J. y Barro, S. (2010). Universitic 2010: Evolución de las TIC en el sistema universitario español 2006 - 2010. Conferencia de Rectores de las Universidades Españolas (CRUE). 